\title{
Detection of apoptosis by the TUNEL technique in clinically localised prostatic cancer before and after combined endocrine therapy
}

\author{
M Colecchia, B Frigo, C Del Boca, A Guardamagna, A Zucchi, D Colloi, O Leopardi
}

\begin{abstract}
Aims-Apoptosis in prostate cancer was evaluated after three months of combined endocrine therapy to investigate the association with tumour grade, tumour stage, and the immunohistochemical detection of p53 and bcl-2 in tumour cells before and after therapy.
\end{abstract}

Methods-Twenty six formalin fixed, paraffin wax embedded core biopsies and corresponding prostatectomy specimens, excised after three months of combined endocrine therapy, were analysed for the presence of apoptotic cells by the terminal deoxynucleotidyl transferase mediated dUTP-biotin nick end labelling (TUNEL) method, and for p53 and bcl-2 overexpression by immunohistochemistry.

Results-All 26 adenocarcinomas were clinically localised at diagnosis. In biopsies performed before combined endocrine therapy, the apoptotic indices varied between $0.09 \%$ and $1.73 \%$, while the tumour grade fell between Gleason score 1 and 8. The mean (SD) apoptotic count pretherapy was $0.71 \%(0.50)$. There was a significant association between elevated apoptotic counts and higher Gleason scores in the biopsies $(p=0.005)$. After three months of therapy, the percentage of apoptotic tumour cells increased independently of tumour stage, while a significant association with Gleason grade was found $(p=0.0018)$ and all the tumours had Gleason scores of $<7$. In eight cases the apoptotic index was more than twice its pretherapy value. The remaining tumours showed less of an increase in the apoptotic index (five cases) or a reduction in the percentage of apoptotic cells. The overall moderate increase in apoptotic index after combined endocrine therapy was not statistically significant $(p=0.8)$. Immunoreactivity to $\mathbf{p} 53$ was absent in all cases, before and after therapy, while a slight increase in the number of cells overexpressing bcl-2 was observed in five of the 13 tumours (38.1\%) with reduced apoptotic indices after therapy.

Conclusions-After three months of combined endocrine treatment a minority of clinically localised prostate neoplasms showed regressive epithelial alterations, associated with an increase in apoptotic tumour cells; an increase in cells overex- pressing bcl-2 was observed in five of the 13 tumours with reduced apoptotic indices.

( Clin Pathol 1997;50:384-388)

Keywords: apoptosis; nick end labelling; prostatic cancer

Combination therapy using a nonsteroidal antiandrogen in association with a luteinising hormone releasing hormone (LHRH) agonist has significant benefits in both advanced and localised prostate cancer; some authors believe neoadjuvant total androgen withdrawal to have some beneficial effects as downstaging, while others reported that the majority of patients with locally advanced prostate carcinoma continue to have extraprostatic carcinoma despite clinical evidence suggestive of downstaging. ${ }^{1-4}$

Histological changes are commonly observed some months after combined endocrine therapy: a decrease in tumour size, vacuolisation of the cytoplasm, and changes in glandular size, the cell proliferation rate, and nuclear and nucleolar size are the most frequent. In vivo studies have identified apoptosis as the molecular event underlying the regression of androgen dependent human prostatic tumours after androgen ablation. ${ }^{5}$ Indeed, the histological appearance of apoptotic bodies is a characteristic early event ${ }^{6}$ that precedes the dramatic reduction in tumour volume observed after three months of combined endocrine therapy. ${ }^{7}$

The present study investigated the frequency of apoptosis, using the terminal deoxynucleotidyl transferase mediated dUTP-biotin nick end labelling (TUNEL) method, in 26 clinically localised prostatic carcinomas obtained by radical prostatectomy after three months of therapy with an LHRH agonist leuprorelin (Enantone Depot, Takeda, Osaka, Japan) and an antiandrogen flutamide (Eulexin, Schering Pharmaceuticals, Milan, Italy). Apoptosis was also evaluated on ultrasound guided core biopsies performed before combined endocrine therapy. Results were analysed for any association with parameters such as Gleason grade, tumour stage, and immunohistochemical investigation of $\mathrm{p} 53$ and bcl-2 overexpression. The aim of our study was to examine the behaviour of these clinically localised prostatic cancers after androgen ablation therapy.

\section{Methods}

Twenty six cases of prostate cancer were selected from the files of the Pathology Department, Ospedale Maggiore di Lodi, Italy. 
Each patient had a clinical stage B cancer and underwent a radical prostatectomy: before surgery all patients had received combined treatment with leuprorelin (3.75 mg per month subcutaneously) and flutamide ( $250 \mathrm{mg}$ orally three times a day) for three months. The histopathological material was 26 ultrasound guided core biopsies performed before the combined endocrine therapy, and the subsequent prostatectomies. Further biopsies were not performed before radical prostatectomy, according to the protocol of neoadjuvant therapy. The biopsies were routinely processed. Grade was assigned using the Gleason system in which five distinct histological patterns are recognised. ${ }^{8}$ The radical prostatectomy specimens were cut into slices $\sim 0.5 \mathrm{~cm}$ thick from the apex to the base of the prostate and then fixed for 24 hours in formalin and embedded in paraffin wax.

\section{LABELLING OF APOPTOTIC CELLS}

The TUNEL method described by Gavrieli et al was used for apoptotic cell labelling. ${ }^{9}$ Thus, after deparaffinisation, sections were incubated with $20 \mu \mathrm{g} / \mathrm{ml}$ proteinase $\mathrm{K}$ for 15 minutes at room temperature and thoroughly washed in sterile distilled water. A rinse in terminal deoxynucleotidyl transferase (TdT) buffer (30 mM Trizma base, pH 7.2, $140 \mathrm{mM}$ sodium cacodylate, $1 \mathrm{mM}$ cobalt chloride) was performed before immersing the slides in a mixture of TdT $(0.3$ units $/ \mu \mathrm{l})$ and biotin dUTP $(0.03 \mathrm{nmols} / \mu \mathrm{l})$ in TdT buffer for 90 minutes at $37^{\circ} \mathrm{C}$ in a humidity chamber. Sections were rinsed in sterile distilled water, immersed in a $2 \%$ aqueous solution of bovine serum albumin (BSA) for 10 minutes at room temperature, rinsed in $0.05 \mathrm{M}$ Tris buffered saline (TBS), $\mathrm{pH} 7.4$, and covered with alkaline phosphatase conjugated streptavidin $(1: 30)$ for 30 minutes at room temperature. After visualisation of the reaction using new fuchsin, slides were slightly counterstained with haematoxylin and mounted.

\section{IMMUNOHISTOCHEMISTRY}

Immunohistochemical studies were performed using the peroxidase conjugated streptavidinbiotin complex method. The mouse monoclonal antibody to p53 protein (Mab D07, Novocastra Laboratories, Newcastle upon Tyne, UK) and the mouse anti-bcl-2 (clone 124 Dakopatts, Glostrup, Denmark), were used, diluted $1: 100$ and $1: 40$, respectively. A threshold of $5 \%$ immunoreactive tumour cells was chosen for the evaluation of p53 overexpression. Immunostaining for bcl-2 was assessed semiquantitatively, based on the proportion of immunoreactive tumour cells; cases were divided into negative $(0 \%)$, and those with $\leqslant 5 \%$ or $>5 \%$ immunoreactive cells.

QUANTITATION OF APOPTOTIC CELLS

The percentages of apoptotic cells (apoptotic index $=$ number of TUNEL positive cells per 100 cells) were determined by counting 1000 tumour cells in randomly chosen areas of biopsy sections on a light microscope at $\times 40$ magnification. On the sections obtained after radical prostatectomy, the apoptotic index was determined by randomly counting 3000 tumour cells per patient.

STATISTICS

The significance of the associations was determined using the $\chi^{2}$ test. The Mantel-Haenszel $\chi^{2}$ test for linear association assessed the significance of trends. A value of $p<0.05$ was considered statistically significant.

\section{Results}

All 26 patients included in the present study were submitted to preoperative combined endocrine therapy. Patients' ages ranged from 59 to 72 years (median 67.3). Tumours were categorised according to the final pathological stage: seven patients were classified as stage pT $2 a$, six as stage pT2b, seven as stage pT2c, four as stage pT3a, and two as stage pT3c. Only one case, at stage pT3a, revealed a metastasis in the pelvic lymphnodal station.

In the haematoxylin and eosin preparations of radical prostatectomy, we found an almost even prevalence of some histopathological changes: non-tumoural prostatic tissue showed cell vacuolisation with smaller nucleoli, and prominence and hyperplasia of basal cells; adenocarcinoma showed decreased intraluminal crystalloids and areas of individual tumour cells, separated by abundant connective tissue, showing inconspicuous nucleoli and cytoplasmic vacuolisation.

For the evaluation of apoptosis, we compared TUNEL treated sections with haematoxylin and eosin stained sections. In each case, cells with the morphological characteristics of apoptosis were identified: chromatin condensation, formation of crescentic caps of condensed chromatin at the nuclear periphery, and formation of apoptotic bodies were visible in both biopsy and prostatectomy specimens, in the intercellular space as well as in the lumen, sometimes ingested by macrophages. As shown in table 1 the apoptotic count in 1000 tumour nuclei evaluated by the TUNEL method on biopsy samples varied considerably (mean (SD) $0.718(0.50)$; range $0.09-1.73 \%)$. The mean apoptotic index in 3000 tumour nuclei after therapy was $0.75(0.51)$ (range $0.097-$ $2.10 \%)$. The overall moderate increase in apoptotic index after combined endocrine therapy was not statistically significant $(p=0.8)$. However, two subgroups of patients with different apoptotic counts were recognised: the first had a twofold, or greater, increase in the apoptotic index after combined endocrine therapy; in the second group, however, the increase in the apoptotic index was less than twofold and in some cases there was even a decrease in the apotopic index after therapy.

HIGH APOPTOPIC INDEX INCREASE GROUP Eight patients showed a twofold, or greater, increase in apoptopic index after combined endocrine therapy. This group contained Gleason grade 4-6 tumours, with a pretherapy apoptopic index oscillating between 0.091 and 
Table 1 Comparison of Gleason grades, apoptopic counts before and after endocrine therapy, and tumour stages in 26 prostatic cancers

\begin{tabular}{llll}
\hline $\begin{array}{l}\text { Gleason } \\
\text { grade }\end{array}$ & $\begin{array}{l}\text { Apoptopic index } \\
\text { (pretreatment) }\end{array}$ & $\begin{array}{c}\text { Apoptopic index } \\
\text { (post-treatment) }\end{array}$ & $\begin{array}{l}\text { Tumour } \\
\text { stage }\end{array}$ \\
\hline 4 & 0.360 & 1.262 & T2cN0 \\
4 & 0.091 & 0.964 & T2cN0 \\
5 & 0.269 & 0.972 & T2cN0 \\
5 & 0.178 & 0.467 & T2bN0 \\
5 & 0.258 & 0.969 & T2bN0 \\
6 & 0.486 & 0.799 & T3aN0 \\
6 & 0.281 & 0.965 & T2cN0 \\
6 & 0.255 & 0.661 & T3a N1 \\
6 & 0.433 & 1.651 & T2bN0 \\
6 & 0.154 & 0.581 & T2aN0 \\
6 & 0.760 & 0.593 & T2aN0 \\
6 & 0.769 & 0.097 & T2aN0 \\
7 & 1.572 & 0.499 & T2bN0 \\
7 & 1.451 & 0.694 & T2cN0 \\
7 & 0.452 & 0.582 & T2aN0 \\
7 & 0.868 & 0.131 & T2aN0 \\
7 & 1.431 & 1.439 & T2aN0 \\
7 & 0.172 & 0.118 & T2aN0 \\
7 & 0.594 & 0.442 & T2cN0 \\
7 & 0.530 & 0.229 & T2bN0 \\
8 & 0.897 & 0.134 & T3aN0 \\
8 & 1.009 & 0.824 & T3aN0 \\
8 & 1.738 & 2.109 & T3cN0 \\
8 & 0.725 & 0.413 & T2bN0 \\
8 & 1.156 & 0.887 & T2cN0 \\
8 & 1.653 & 1.448 & T3cN0 \\
Total (mean $($ SD)) (n=26) & & \\
& $0.718(0.505)$ & $0.751(0.515)$ & \\
\hline & & &
\end{tabular}

0.433 (mean $0.25(0.11)$ ), and post-therapy apoptopic indices of $0.46-1.65$ (mean 0.96 $(0.34)$ ). All tumours were localised to the prostate gland.

APOPTOPIC INDEX DECREASE/LOW APOPTOPIC INDEX INCREASE GROUP

After combined endocrine treatment five tumours showed less than a twofold increase in the apoptopic index, while 13 showed a decrease. Before therapy, all tumours had Gleason scores of 6-8, various tumoural patterns (cribriform, solid, or trabecular), and a particularly high apoptopic index before therapy (0.17-1.73, mean $0.91(0.48))$. The apoptopic index after therapy varied between 0.097 and 2.109 (mean $0.64(0.54)$ ).

All biopsies were negative for $\mathrm{p} 53$, while bcl-2 immunostaining detected the protein in a few cells in 10 pretherapy biopsies. After therapy, p53 immunoreactivity was not changed, while the bcl-2 immunostaining was conspicuously increased in five tumours, all belonging to the low apoptotic index group; however, the proportion of immunoreactive tumour cells did not exceed $5 \%$ in any of the cases.

The Mantel-Haenszel test for linear association showed a highly significant association of Gleason grading with apoptotic counts ( $p=0.0018$ ), with a Gleason grade of $<7$ significantly found more frequently in the high apoptopic index increase group. The linear association between apoptopic index before therapy and Gleason grade was highly significant as assessed by the Mantel-Haenszel test $(p=0.0051)$, with well differentiated tumours showing lower indices. This significant linear association between a low apoptopic index and a higher Gleason grade was not present after endocrine therapy $(p=0.78)$. There was no significant predictability of tumoural behaviour according to the age of patient. Low apoptotic counts before endocrine therapy were significantly associated with an increase in apoptosis after treatment (Mantel-Haenszsel test for linear association, $\mathrm{p}=0.001)$.

\section{Discussion}

Previous work concerning the evaluation of apoptosis in vitro has shown that the time window during which the morphological changes due to programmed cell death occur is relatively short (between 30 minutes and two hours); thus, many cells in the early stages of apoptosis may escape detection in vivo. ${ }^{10}$ The TUNEL method is based on direct, specific, in situ labelling of DNA breaks in the nuclei. Gorczyca and coworkers applied this method to human solid tumours sampled by fine needle biopsy, and reported that the apoptotic phase characterised by the presence of DNA strand breaks, which precedes the gross changes in cell morphology, is very long. ${ }^{11}$ The TUNEL technique labels the small $(<2 \mu \mathrm{m})$ apoptotic bodies as well as the classical crescentic caps of condensed chromatin at the nuclear periphery, ${ }^{12}$ thus avoiding confusion with other cells that have condensed chromatin, such as telophase mitotic cells or polymorphic granulocytes (fig 1). Furthermore, this technique allows one to differentiate the condensed chromatin of apoptotic nuclei from the nuclei of lymphocytes that diffusely infiltrate prostate adenocarcinomas after combined endocrine therapy. Therefore, according to Gavrieli and coworkers, ${ }^{9}$ by detecting DNA fragmentation that occurs before the appearance of morphological features, the TUNEL method is a highly sensitive technique that "enables a determination of the actual fraction of cells undergoing programmed cell death". The presence of apoptosis after combined endocrine therapy is a well known phenomenon, ${ }^{7}{ }^{13}$ but the focality of apoptosis evaluated in some series by nuclear morphology alone, suggested that the majority of residual carcinoma cells are resistant to apoptosis following three months of combined therapy. As Armas et al postulated, data concerning the apoptopic index could be underestimated, either because of the possible completion of hormone regulated apoptosis after three months of therapy, the heterogeneity of androgen sensitivity among subpopulations of tumour cells, or the difficulty of detecting apoptosis by the observation of nuclear morphology in haematoxylin and eosin stained sections.

Using the TUNEL method on 26 biopsies with prostate adenocarcinoma, we found higher percentages of apoptotic bodies than Aihara and colleagues did $^{15}$ who evaluated 39 untreated cases of prostate cancer by nuclear morphology in haematoxylin and eosin stained sections. Indeed, while Aihara et al (who reported only the principal Gleason score) found apoptotic bodies ranging from $0.05 \%$ in Gleason grade 1 tumours, to $0.66 \%$ in Gleason grade 5 tumours, in the present study, in which the Gleason sum was used, the apoptopic index before combined therapy ranged from $0.09 \%$ in tumours with a Gleason sum of $4(2+2)$, to 


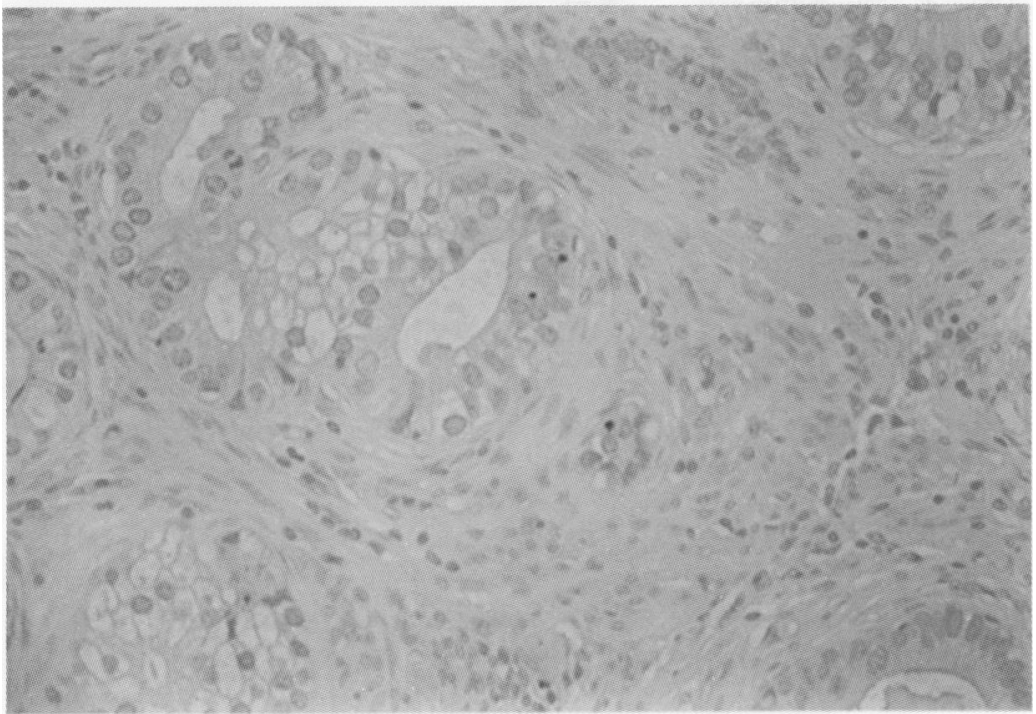

Figure 1 Apoptotic bodies detected within tumoural glands in prostatic cancer after combined endocrine therapy (TUNEL technique).

$1.73 \%$ in tumours with a Gleason sum of 8 $(4+4)$. However, a clear positive linear association between the apoptopic index and the Gleason score came out in both papers. Gaffney assessed apoptosis semiquantitatively by light microscopy on routine haematoxylin and eosin stained sections in 15 high grade adenocarcinomas of the prostate, and reported a mean apoptopic index of $1.7 \%$ (range $0.2-2.4 \%$ ) in nine solid undifferentiated carcinomas. He found higher counts in more unusual high grade neoplasms; three small cell undifferentiated prostate cancers (mean apoptopic index $25.2 \%$ ) and three transitional carcinomas of the bladder involving the prostate (mean apoptopic index 5.5\%). ${ }^{16}$

Our series of prostate cancers showed a variable behaviour after therapy: eight patients $(30.8 \%)$ showed a considerable increase in apoptopic index (twofold or more); all had well, or moderately, differentiated p 53 negative tumours with a Gleason sum of $<6$ and were stage pT2 $(a, b$, or c) prostate cancers. Androgen withdrawal had different effects in the remaining 18 tumours, with a decrease or minimal increase in apoptopic index. These findings are similar to the short term effects reported after castration therapy in 18 core biopsies of prostate cancer by Westin et $a l,{ }^{17}$ who reported that one-third of the tumours had a twofold increase in apoptopic index after one week. They were WHO grade 1 or 2 tumours, and were p53, c-myc, and bcl-2 negative, or contained some bcl- 2 or c-myc positive tumour cells.

In our study, all 26 clinically stage B prostate cancers were p53 negative as in other series of clinically localised prostate adenocarcinomas. ${ }^{18-22}$ The absence of immunohistochemically p53 positive cases avoided the evaluation of the different behaviour of p53 negative and positive prostate cancers. According to Westin and coworkers, ${ }^{17}$ the $\mathrm{p} 53$ positive cancers are all responsive to castration therapy, while variable results were reported for p53 negative tumours.
Bcl-2 expression increases in prostatic tumours two or three months after combined endocrine therapy ${ }^{23}$; we have observed a relevant increase in five tumours with decreased apoptopic indices after therapy ( 5 of $13,38.1 \%)$. As Westin et al reported, ${ }^{17}$ it could be important to evaluate the number of bcl-2 positive cells before therapy, to assess the resistance to combined endocrine therapy and the decrease in apoptopic index in bcl-2 positive tumours. In fact, initiation of apoptosis may occur as a consequence of conflicting growth signals and "a hierarchical relationship between bcl-2, p53, c-myc and other genes indicate a complex pattern of regulation of programmed cell death". ${ }^{24}$

In conclusion, the TUNEL method is a useful tool for the evaluation of different apoptotic counts in prostatic cancer after combined endocrine therapy, particularly as it demonstrated differences between the behaviour of low Gleason grade $(\leqslant 6)$ cancers, confined to the organ, and confined or locally advanced higher Gleason grade $(\geqslant 6)$ cancers.

We are grateful to Professor R Montironi of the Department of Pathology, University of Ancona, Italy, who kindly advised us on the preparation of this manuscript; we are also grateful to Miss Erika Prian for technical assistance.

1 Labrie F, Belanger A, Simard J, Labrie C, Dupont A. Combination therapy for prostate cancer. Endocrine and biologic basis of its choice as new standard firstline therapy. Cancer 1993;71:1059-67.

2 Fair WR, Aprikian A, Sogani P, Reuter V, Whitmore WF. The role of neoadjuvant hormonal manipulation in localized prostatic cancer. Cancer 1993;71(Suppl): 1031-8.

3 Aprikian AG, Fair WR, Reuter VE, Sogani P, Herr H, Russo $\mathrm{P}$, et al. Experience with neoadjuvant diethylstilboestrol and $\mathrm{P}$, et al. Experience with neoadjuvant diethylstilboestrol and radical prostatectomy in patients with 1 .
prostate cancer. $B r \mathcal{F}$ Urol 1994;74:630-6.

4 Graham SD, Keane TE, Petros, Sanders WH. Perioperative hormonal therapy in locally advanced adenocarcinoma of the prostate. Cancer 1995;75:1969-71.

5 Kyprianou N, English HF, Isaacs JT. Programmed cell death during regression of PC-82 human prostate cancer following androgen ablation. Cancer Res 1990;50:3748-53.

6 Kyprianou N. Apoptosis: therapeutic significance in the treatment of androgen-dependent and androgentreatment of androgen-dependent and androgenindepen

7 Armas OA, Aprikian AG, Melamed J, Cordon-Cardo C, Cohen DW, Erlandson R, et al. Clinical and pathobiologica effects of neoadjuvant total androgen ablation therapy on clinically localized prostatic adenocarcinoma. Am $\mathcal{f}$ Sur Pathol 1994;18:979-91.

8 Gleason DF, Mellinger GT and the VACURG. Prediction of prognosis for prostatic adenocarcinoma by combined histologic grading and clinical staging. $\mathcal{F}$ Urol 1974;111:5864.

9 Gavrieli Y, Sherman Y, Ben-Sasson SA. Identification of programmed cell death in situ via specific labeling of nuclear DNA fragmentation. F Cell Biol 1992;119:493501

10 Bursch W, Oberhammer F, Schulte-Herman R. Cell death by apoptosis and its protective role against disease. Trends Pharmacol Sci 1992;13:245-51.

11 Gorczyca W, Tuziak T, Kram A, Melamed MR, Darzynkiewicz Z. Detection of apoptosis-associated DNA strand breaks in fine-needle aspiration biopsies by in situ end breaks in fine-nedle aspira Cytometry 1994:15:169-75.

12 Gorczyca W, Gong J, Darzynkiewicz Z. Detection of DNA strand breaks in individual apoptotic cells by the in situ terstrand breaks in individual apoptotic cells by the in situ terminal deoxynucleotidyl transferase

13 Harriss DR, Savill J. Apoptosis and the prostate. $B r f$ Urol 1995;75(Suppl 1):27-33.

14 Montironi R, Magi-Galluzzi C, Muzzonigro G, Prete E, Polito M, Fabris G. Effects of combination endocrine treatment on normal prostate, prostatic intraepithelial neoplasia and prostatic adenocarcinoma. $f$ Clin Patho 1994;47:906-13.

15 Aihara M, Truong LD, Dunn JK, Wheeler TM, Scardino PT, Thompson TC. Frequency of apoptotic bodies positively correlates with Gleason grade in prostate cancer. Hum Pathol 1994;25:797-801.

16 Gaffney EF. The extent of apoptosis in different types of high grade prostatic carcinoma. Histopathology 1994;15: 169-75. 
17 Westin P, Stattin P, Damber J-E, Bergh A. Castration therapy rapidly induces apoptosis in a minority and therapy rapidly induces apoptosis in a minority and decreases cell proliferation in a majority of
tumours. Am $\mathcal{f}$ Pathol 1995;146:1368-75.

18 Mellon K, Thompson S, Charlton RG, Marsh C, Robinson $\mathrm{M}$, Lane DP, et al. p53, c-erb B-2 and the epidermal growt factor receptor in the benign and malignant prostate. $\mathcal{F}$ Uro 1992;147:496-9.

19 Hall MC, Navone NM, Troncoso P, Pollack A, Zagars GK von Eschenbach AC, et al. Frequency and characterization of p53 mutations in clinically localized prostate cancer. Urology 1995;45:470-5.

20 Aprikian AG, Sarkis AS, Fair WR, Zhang Z-F, Fuks Z, Cordon-Cardo $C$. Immunohistochemical determination of p53 protein nuclear accumulation in prostatic adenocarcnioma. 7 Urol 1994;151:1276-80.
21 Ittmann $M$, Wieczorek $R$, Heller $P$, Dave A, Provet J, Krolewski J. Alterations in the p53 and $\mathrm{mdm}-2$ genes are infrequent in clinically localized, stage B prostate adenocarinfrequent in clinically localized, stage B
cinomas. Am f Pathol 1994;145:287-93.

22 Navone NM, Troncoso P, Pisters LL, Goodrow TL, Palmer $\mathrm{JL}$, Nichols WW, et al. $\mathrm{p} 53$ protein accumulation and gene mutation in the progression of human prostate carcinoma. 7 Natl Cancer Inst 1993;85:1657-69.

23 Colombel M, Symmans F, Gil S, O'Toole KM, Chopin D, Benson $M$, et al. Detection of the apoptosis-suppressing oncoprotein bcl-2 in hormone refractory-human prostate cancers. Am f Pathol 1993;8:390-400.

24 Stewart BW. Mechanisms of apoptosis: integration of genetic, biochemical, and cellular indicators. 7 Natl Cancer Inst 1994;86:1286-96. 